\title{
Functional Reorganization of the Brain in Humans Following Spinal Cord Injury: Evidence for Underlying Changes in Cortical Anatomy
}

\author{
Luke A. Henderson, ${ }^{1}$ Sylvia M. Gustin, ${ }^{1,2}$ Paul M. Macey, ${ }^{3}$ Paul J. Wrigley, ${ }^{2}$ and Philip J. Siddall ${ }^{2}$ \\ ${ }^{1}$ Department of Anatomy and Histology, University of Sydney, Sydney, New South Wales 2006, Australia, ${ }^{2}$ Pain Management Research Institute, Division \\ of the Kolling Institute of Medical Research, University of Sydney, Royal North Shore Hospital, St. Leonards, New South Wales 2065, Australia, and \\ ${ }^{3}$ Department of Neurobiology, University of California, Los Angeles, Los Angeles, California 90024
}

Loss of somatosensory drive results in functional reorganization of the primary somatosensory cortex (SI). While the phenomenon of functional cortical reorganization is well established, it remains unknown whether in humans, functional reorganization results from changes in brain anatomy, or simply reflects an unmasking of already existing dormant synapses. In 20 subjects with complete thoracic spinal cord injuries (SCIs) and 23 controls, we used functional and structural magnetic resonance imaging to determine whether SI reorganization was associated with changes in SI anatomy. SCI resulted in a significant SI reorganization, with the little finger representation moving medially toward the lower body representation (i.e., area of sensory loss). Furthermore, although SCI was associated with gray matter volume loss in the lower body representation, this loss was minimized as reorganization increased. That is, the greater the medial shift in little finger representation, the greater the gray matter preservation in the lower body representation. In addition, in the region of greatest SI reorganization (little finger), fractional anisotropy was correlated with SI reorganization. That is, as SI reorganization increased, the extent of aligned structures decreased. Finally, although thalamocortical fibers remained unchanged, the ease and direction of water movement within the little finger representation was altered, being directed more toward the midline in SCI subjects. These data show that SI reorganization following SCI is associated with changes in SI anatomy and provide compelling evidence that SI reorganization in humans results from the growth of new lateral connections, and not simply from the unmasking of already existing lateral connections.

\section{Introduction}

It is well established that a loss of afferent sensory drive is followed by functional reorganization of the primary somatosensory cortex (SI) (Merzenich et al., 1983, 1984; Allard et al., 1991; Pons et al., 1991; Flor et al., 1995; Wrigley et al., 2009). That is, the area of SI deprived of its normal inputs is activated by sensory stimulation of the surrounding intact body regions. Despite the well established phenomenon of cortical reorganization, it remains unknown whether in humans, this functional reorganization results from physical changes in brain anatomy, such as dendritic sprouting, or simply reflects an unmasking of already existing dormant synapses. Although it has recently been reported in humans that SI reorganization can occur within hours of limb anesthesia, supporting the existence of dormant synapses (Björkman et al., 2009), it remains unknown whether such an "unmasking" is responsible for the large SI reorganization that occurs following limb amputation or spinal cord injury (SCI).

Received May 27, 2010; revised 0ct. 25, 2010; accepted Nov. 22, 2010.

This work was supported by grants awarded by the New South Wales Office for Science and Medical Research. We thank Kirsten Moffat for help in all imaging procedures. Scans were performed at the Prince of Wales Imaging Centre.

Correspondence should be addressed to Luke A. Henderson at the above address. E-mail: lukeh@anatomy.usyd.edu.au.

DOI:10.1523/JNEUROSCI.2717-10.2011

Copyright $(2011$ the authors $\quad 0270-6474 / 11 / 312630-08 \$ 15.00 / 0$
Over 10 years ago, Florence et al. (1998) investigated thalamocortical projection patterns in macaque monkeys with longstanding arm injuries including amputation. They found that although microelectrode recordings revealed massive reorganization of the affected limb SI representation, subsequent tract tracing revealed normal thalamocortical connections, but significantly expanded lateral connections. They suggested that the growth of intracortical, not thalamocortical connections, was responsible for the observed SI reorganization. Furthermore, nearly two decades ago, Pons et al. (1991) also reported a substantial degree of SI reorganization in monkeys with unilateral dorsal rhizotomies of all cervical spinal segments, with the representation of the face expanding by up to $14 \mathrm{~mm}$. The authors suggested that the physical size of this reorganization was too great to be accounted for by solely the unmasking of latent connections and overlap of thalamocortical projections and must involve the growth of lateral connections (Garraghty and Sur, 1990; Rausell and Jones, 1995).

Despite evidence in experimental animals that cortical reorganization can involve physical changes in brain anatomy, it remains unknown whether in humans, SI reorganization involves changes in cortical anatomy. It is important to understand the precise nature of the underlying mechanisms of cortical organization in humans as anatomical changes are likely to be more resistant to treatments aimed at reversal. The aim of this investi- 
Table 1. Spinal cord injury subject characteristics

\begin{tabular}{|c|c|c|c|c|c|c|c|c|c|c|}
\hline \multirow[b]{2}{*}{ Subject } & \multirow[b]{2}{*}{ Age } & \multirow{2}{*}{$\begin{array}{l}\text { Years } \\
\text { since } \\
\text { injury }\end{array}$} & \multirow{2}{*}{$\begin{array}{l}\text { Neurological } \\
\text { level of SCl }\end{array}$} & \multirow[b]{2}{*}{ AIS } & \multicolumn{2}{|c|}{ Motor level } & \multicolumn{2}{|c|}{$\begin{array}{l}\text { Sensory } \\
\text { level }\end{array}$} & \multicolumn{2}{|c|}{$\begin{array}{l}\text { Sensory } \\
\text { ZPP }\end{array}$} \\
\hline & & & & & Right & Left & Right & Left & Right & Left \\
\hline 1 & 40 & 5 & T7 & $A$ & $\mathrm{~T} 1$ & $\mathrm{~T} 1$ & T7 & T8 & T12 & T12 \\
\hline 2 & 54 & 27 & $\mathrm{~T} 2$ & $A$ & T1 & $\mathrm{T} 1$ & T5 & $\mathrm{T} 2$ & T12 & L2 \\
\hline 3 & 29 & 4 & $\mathrm{~T} 5$ & $A$ & $\mathrm{~T} 1$ & $\mathrm{~T} 1$ & T6 & T5 & L1 & L1 \\
\hline 4 & 34 & 8 & T4 & $A$ & $\mathrm{~T} 1$ & T1 & T4 & T4 & T6 & T5 \\
\hline 5 & 57 & 32 & T4 & $A$ & $\mathrm{~T} 1$ & T1 & T4 & T4 & T5 & T6 \\
\hline 6 & 41 & 14 & T3 & $A$ & $\mathrm{~T} 1$ & T1 & T4 & T3 & T6 & T6 \\
\hline 7 & 27 & 2 & T1 & $A$ & T1 & T1 & $\mathrm{T} 1$ & T1 & T5 & T4 \\
\hline 8 & 51 & 3 & T9 & $A$ & T1 & L2 & T9 & T9 & S2 & S2 \\
\hline 9 & 63 & 5 & $\mathrm{~T} 10$ & $A$ & T1 & T1 & T11 & $\mathrm{T} 10$ & $\mathrm{~T} 12$ & T12 \\
\hline 10 & 54 & 37 & T3 & $A$ & T1 & T1 & T3 & T4 & T6 & T5 \\
\hline 11 & 43 & 21 & T8 & $A$ & T1 & T1 & T8 & T9 & T10 & T10 \\
\hline 12 & 26 & 7 & $\mathrm{~T} 10$ & $A$ & T1 & T1 & T10 & $\mathrm{T} 10$ & L1 & T12 \\
\hline 13 & 44 & 27 & T5 & $A$ & T1 & $\mathrm{T} 1$ & T6 & $\mathrm{T} 5$ & L3 & S2 \\
\hline 14 & 52 & 9 & T3 & A & T1 & $\mathrm{T} 1$ & T3 & T3 & T4 & T4 \\
\hline 15 & 40 & 16 & T3 & $A$ & $\mathrm{~T} 1$ & $\mathrm{~T} 1$ & T3 & T3 & T4 & T4 \\
\hline 16 & 32 & 7 & T3 & $A$ & T1 & $\mathrm{T} 1$ & T3 & T3 & T5 & T7 \\
\hline 17 & 22 & 3 & T5 & $A$ & T1 & T1 & T5 & T5 & T8 & T11 \\
\hline 18 & 37 & 11 & T6 & $A$ & T1 & $\mathrm{T} 1$ & T6 & T9 & $\mathrm{T} 11$ & $\mathrm{~T} 12$ \\
\hline 19 & 53 & 17 & T6 & $A$ & T1 & T1 & T6 & T6 & T11 & $\mathrm{T} 12$ \\
\hline 20 & 32 & 3 & $\mathrm{~T} 5$ & $A$ & T1 & $\mathrm{T} 1$ & $\mathrm{~T} 5$ & $\mathrm{~T} 5$ & T12 & L3 \\
\hline Mean ( \pm SEM) & $38(3)$ & $12(3)$ & $\mathrm{T} 5(1)$ & & & & & & & \\
\hline
\end{tabular}

Neurological level of SCl, The most caudal segment of the spinal cord with normal sensory and motor function on both sides of the body; AIS A, an absence of sensory and motor function, which includes the lowest sacral segments; motor/sensory level, the most caudal segment of the spinal cord with normal motor/sensory function; sensory zone of partial preservation (ZPP), the most caudal sensory dermatome that remains partially innervated. termined using the American Spinal Injury Association (ASIA) Impairment Scale (AIS) (Marino et al., 2003). Each subject was examined by a clinician (P.J.W.) in the research group. The standardized ASIA examination protocol was used to determine the most caudal level of the spinal cord with normal sensory and motor function on both sides of the body. An injury was termed complete when there was an absence of sensory and motor function, which included the lowest sacral segments.

To assess whether SCI resulted in increased fine upper limb movements, each SCI subject was asked to compare the average use of their hands for detailed task before and following their SCI. Each SCI subject was asked the following questions: (1) "Compared to before your SCI, I use my hands now: very rarely, rarely, in the same manner, more frequently, or much more frequently," and (2) "Compared to before your SCI, has the use of your hands for detailed tasks changed (e.g., playing guitar or piano): I do not use my hands for more for detailed tasks, I sometimes use my hands for more detailed tasks, I frequently use my hands for detailed tasks." Informed written consent was obtained for all procedures and the study was approved by the Northern Sydney and Central Coast Area Health Service Institutional Human Research Ethics Committees.

\section{MRI scans}

Subjects lay supine on the bed of a 3T MRI scanner (Philips, Intera) with their head immobilized in a tight-fitting head coil. Four continuous series of 130 gradient echo, echo-planar images, using blood oxygen level-dependent contrast and covering the entire brain were collected. Each functional MRI (fMRI) series was collected over a period of $390 \mathrm{~s}$ (57 axial slices, repetition time $=4 \mathrm{~s}$, echo time $=30 \mathrm{~ms}$, flip angle $=90^{\circ}$, field of view $=250 \mathrm{~mm}$, raw voxel size $=1.95 \times 1.62 \times 3.3 \mathrm{~mm}$ ). A 10 volume baseline period was followed by a 10 volume period during which either the right corner of the mouth, the pad of the right thumb, the pad of the right little finger, or the pad of the right big toe was continuously brushed with a fine plastic brush at $\sim 2$ strokes/s. This was repeated another 5 times for a total of 6 baseline and 6 brushing periods.

In addition, using a single-shot multisection spin-echo echo-planar pulse sequence (repetition time $=8788 \mathrm{~ms}$; flip angle $=90^{\circ}$, matrix size $=112 \times 112$, field of view $=224 \times 224$ $\mathrm{mm}$, slice thickness $=2.5 \mathrm{~mm}, 55$ axial slices), four high-resolution DTI image sets covering the entire brain were collected. For each slice, diffusion gradients were applied along 32 independent orientations with $b=1000 \mathrm{~s} / \mathrm{mm}^{2}$ after the acquisition of $b=0 \mathrm{~s} / \mathrm{mm}^{2}\left(b_{0}\right)$ images. Four DTI series were collected separately for subsequent averaging.

\section{MRI analysis}

fMRI. Using SPM5 (Friston et al., 1995), fMRI images were realigned and spatially normalized to the MNI template, global signal changes were removed using the detrending method described by Macey et al. (2004), and the images were spatially smoothed ( $6 \mathrm{~mm}$ FWHM). Signal intensity increases were assessed using a gation is to use voxel-based morphometry of T1-weighted and diffusion tensor images (DTIs) to determine whether the SI reorganization that occurs following SCI is associated with changes in cortical anatomy. Furthermore, using fiber-tracking techniques, we aim to determine whether changes in cortical anatomy reflect alterations in the properties of thalamocortical axons or changes within the cortical gray matter itself.

\section{Materials and Methods}

\section{Subjects}

Twenty subjects with established complete thoracic SCI (18 males, 2 females; aged 22-63 years) and 23 healthy control subjects without SCI (20 males; 3 females; aged 23-60 years) were recruited for the study (Table 1). For the SCI subjects, the neurological level of injury was de- repeated box-car model convolved with a conical hemodynamic response function. The maximally activated voxel in contralateral (left) SI was determined in each individual subject during each of the four brushing paradigms and the Euclidean distance between the maximally activated voxel and an anatomical marker (dorsal point where the central sulcus meets the midline) was determined and compared between the $\mathrm{SCI}$ and control groups (two-tailed $t$ test) (for details, see Wrigley et al., 2009). The degree of cortical reorganization of the little finger was displayed as absolute changes compared to healthy controls (difference of the euclidean distance between controls and SCI).

In no SCI subject did brushing of the big toe evoke a significant change in SI signal intensity. In 15 of the remaining $152 \mathrm{fMRI}$ scans, brushing did not result in any significantly activated voxels within the contralateral SI (3 lip; 0 thumb; 9 little finger; 3 big toe). 
For display purposes and for subsequent DTI tractography, clusters containing the highest activated 20 contiguous voxels within the left SI and the left thalamus during each brushing paradigm were derived and overlaid onto an individual's T1-weighted anatomical image. To take into consideration the bilateral nature of spinal cord injury, these clusters were reflected across the midline for display on the right SI. The percentage change in signal intensity (relative to the first 10 volumes) was then calculated from this cluster for each individual subject and these were then averaged to create a mean \pm SEM signal intensity change time-trend.

VBM of T1-weighted images. Using SPM, the three T1-weighted images from each subject were coregistered and averaged. The averaged image was bias corrected using the SPM5 unified segmentation (Ashburner and Friston, 2005). The bias-corrected images were segmented and spatially normalized using a second pass of the unified segmentation algorithm. The results of the segmentation and spatial normalization were whole-brain "maps" of gray matter probabilities, spatially normalized into the Montreal Neurological Institute (MNI) template space, and "modulated" by the volume changes due to the normalization (Ashburner and Friston, 2000). The normalized, modulated gray matter images were smoothed (full width at half maximum $=6 \mathrm{~mm}$ ). A voxel-by-voxel analysis was performed to search for gray matter volumes within SI that were significantly correlated with the degree of cortical reorganization in SCI subjects. The degree of reorganization was measured by the Euclidean distances observed during brushing of the little finger as changes in this area were most pronounced (random effects, uncorrected $p<0.001$, age and gender as nuisance variables). Significant gray matter volumes were then overlaid onto an individual's T1-weighted anatomical image for visualization.

DTI-indices. The DTI images were processed using SPM5 and Matlab-based custom software. The diffusion-weighted images were motion corrected, coregistered to one another, and averaged to increase signal-to-noise ratio. Using the 32 directions and $b_{0}$ images, the diffusion tensor was then calculated using the method proposed by Basser and Pierpaoli (1996). Once the elements of diffusion tensor were calculated, fractional anisotropy (FA) maps were derived and the images spatially normalized and smoothed $(6 \mathrm{~mm}$ FWHM). A voxel-by-voxel analysis was performed to search for FA values within SI that were significantly correlated with the degree of cortical reorganization in SCI subjects, i.e., using the Euclidean distances observed during brushing of the little finger (random effects, uncorrected $p<0.001$, age and gender as nuisance variables). Significant FA clusters were then overlaid onto an individual's T1-weighted anatomical image. For each significant cluster, the relationship between FA and time since injury was also assessed.

DTI-fiber tracking. Tractography was performed using mrDiffusion software (Dougherty et al., 2005), based on the diffusion tensor calculated as described above. Fibers were tracked between the thalamic region activated during brushing of the lip, little finger, thumb, and toe and the SI region in which MD was correlated with SI reorganization. For each subject, these clusters were "un-normalized" into their native space and the output fibers tracked from this seeding cluster using a minimum FA value of 0.15 and a maximum turning angle of $30^{\circ}$. These fibers were then clipped so that any fibers caudal to the thalamus were removed and the resulting fibers were then normalized into MNI space, binarized, and added to the output fibers from other subjects to provide an indication of the frequency of each fiber bundle in all 40 subjects (controls $n=23$; SCI $n=17$ ). The mean FA values of the fibers were calculated in each subject and averaged for the control and SCI groups, and differences between the groups were determined (two-sample $t$ tests, two tailed, $p<0.05$ ).

DTI-principal eigenvector analysis. For selected areas in SI showing functional reorganization, the directional nature of structure was indicated with a vector on a polar plot, corresponding to the direction of the principal eigenvector of the diffusion tensor (see above, DTI-indices). The vector was scaled by the fractional anisotropy, so that regions with little directional organization of structure would show small vectors. This vector indicates the predominant direction of water diffusion, and therefore is an indirect measure of axonal direction. We calculated the mean direction 


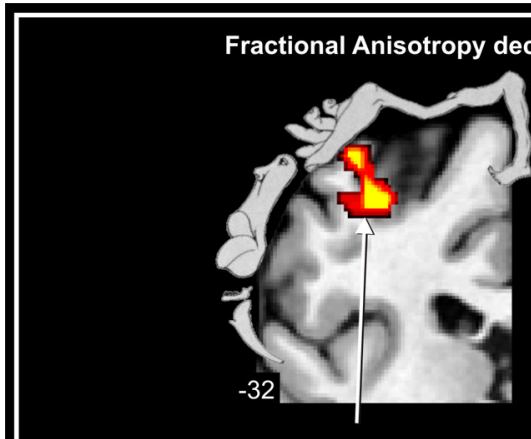

Left SI

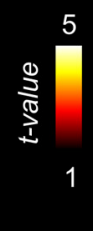

1

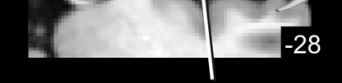

Right SI
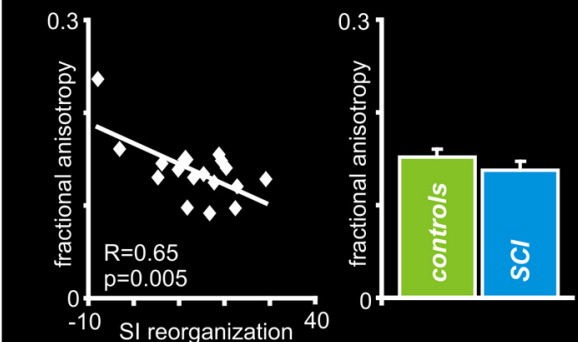

क

(medial displacement in $\mathrm{mm}$ )

Fractional Anisotropy decrease is located in SI region representing the hand
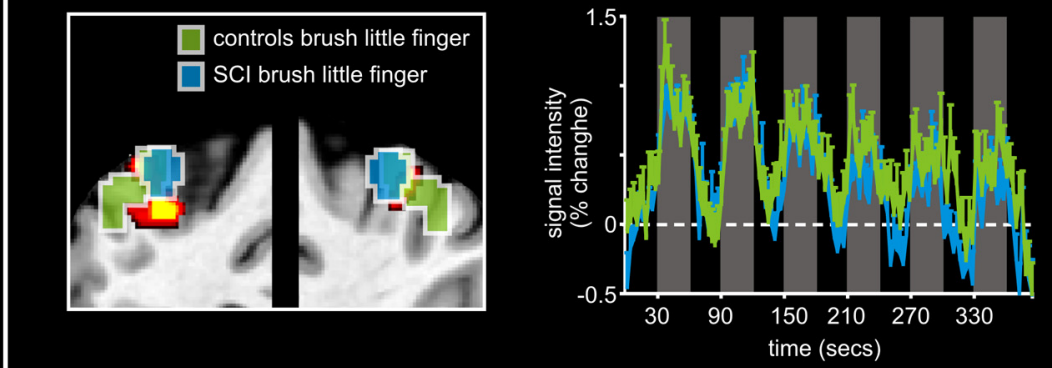

Figure 3. Top, Regions of the $\mathrm{SI}$ in which the degree of functional reorganization is significantly correlated with fractional anisotropy (hot color scale) in SCl subjects. The value at the bottom of each coronal section indicates the slice location in Montreal Neurological Institute space. Below are plots of fractional anisotropy against the degree of SI reorganization (displayed as difference of the euclidean distance between controls and SCI), and average ( \pm SEM) fractional anisotropy in SCI (blue) and control (green) subjects. Note that fractional anisotropy decreases in a discrete region of $\mathrm{SI}$ as medial displacement of the little finger representation increases. Bottom, Region of SI that is activated by innocuous brushing the little finger in control (green) and SCI (blue) subjects. Note that this region overlaps the region of fractional anisotropy change in $\mathrm{SCl}$ subjects. To the right is a plot of the mean percentage change in signal intensity during brushing of the little finger in control and $\mathrm{SCl}$ subjects. The vertical gray bars indicate the brushing periods. Note that brushing the little finger evokes signal intensity increases in both $\mathrm{SCl}$ and control subjects.

across voxels in regions that showed the greatest structural differences (see above, DTI-indices) to better understand the nature of those structural changes. We also indicated variation by displaying vectors of the mean \pm the mean absolute deviation (in lieu of SD, which is complex for vectors).

\section{Results}

\section{Cortical reorganization}

All subjects had complete (AIS A; profound loss of sensation and motor control below the injury level with absence in the sacral segments) thoracic spinal cord injuries. Fourteen of the twenty SCI subjects stated that they did not use their upper limbs to perform fine manipulation more frequently following their SCI than before their injury. Three SCI patients stated they used their upper limbs more for detailed tasks, and the remaining three reported they used their upper limbs for a lot more following their SCI.

The sensory loss was associated with a significant SI reorganization. That is, the SI representations of the thumb and little finger were displaced medially in SCI subjects compared to control subjects. Mean ( \pm SEM) euclidean distance for the lip was as follows: controls $70.8 \pm 0.8 \mathrm{~mm}$, SCI $69.5 \pm 1.5 \mathrm{~mm}$; for the thumb: controls $65.8 \pm 1.3 \mathrm{~mm}$, SCI $59.3 \pm 2.6 \mathrm{~mm}(p<$ $0.05)$; and for the little finger: controls $62.3 \pm 2.0 \mathrm{~mm}$, SCI $48.8 \pm 2.2 \mathrm{~mm}(p<$ 0.0005) (Fig. 1). Therefore, compared to controls, in SCI subjects, the thumb activation was displaced $\sim 7 \mathrm{~mm}$ and the little finger activation $13 \mathrm{~mm}$ toward the midline. Although the degree of SI reorganization was not significantly correlated with the injury level $(r=0.13 ; p>0.05)$, it was significantly correlated with time since injury $(r=-0.51 ; p<0.05)$ and age $(r=$ $-0.57 ; p<0.05)$. That is, the greater the age and time since injury, the greater the medial displacement of the little finger.

\section{Anatomical changes in SI region of sensory loss (lower body)}

Complete thoracic SCI was associated with a significant reduction in gray matter volume in the region of SI that would normally represent the lower body [mean \pm SEM (prob $\times$ vol): right SI: controls $0.45 \pm 0.05$; SCI $0.33 \pm 0.02$; left SI: controls $0.39 \pm 0.07$; SCI $0.26 \pm 0.03$ ] (Fig. 2). Although SCI was associated with a significant decrease in gray matter volume in the SI region of sensory loss, the magnitude of this gray matter decrease was minimized with increased medial displacement of the surrounding SI representations. That is, the greater the invasion of surrounding SI representations (greater medial displacement), the less gray matter loss (increased gray matter volume) within the leg area of SI (left SI: $r=0.71, p=0.001$; right SI: $r=0.62$, $p=0.008)$. Gray matter loss was not correlated with the injury level $(p>0.05$; left SI: $r=0.05$; right SI: $r=0.06)$. Furthermore, MD values within the area of gray matter loss were not significantly different between controls and SCI subjects [mean \pm SEM $\left(\mathrm{mm}^{2} / \mathrm{s} \times\right.$ $\left.10^{-3}\right)$ : right SI: controls $1.13 \pm 0.04$; SCI $1.17 \pm 0.04$; left SI: controls $1.61 \pm 0.05$; SCI $1.73 \pm 0.06$ ] and were not correlated with the degree of SI reorganization.

Comparison of the locations of SI activations during innocuous brushing of the big toe in control subjects and the region of gray matter change in SCI subjects confirmed that the gray matter loss was located in the region of SI previously representing the lower body. Furthermore, brushing the big toe in SCI subjects did not evoke a significant signal intensity increase within SI.

\section{Anatomical changes in SI region undergoing reorganization (little finger)}

In addition to change in gray matter volume, in SCI subjects, the degree of SI reorganization was significantly correlated with FA in the region of SI representing the hand (Fig. 3). That is, the greater 
the little finger activation was displaced medially compared to controls (greater SI reorganization), the lower the FA within the hand area of SI (left SI: $r=0.65, p=$ 0.005 ; right SI: $r=0.82, p=0.00006)$. This region did not display a significant FA difference in SCI compared with control subjects (mean \pm SEM: right SI: controls $0.227 \pm 0.009$; SCI $0.209 \pm 0.008$; left SI: controls $0.145 \pm 0.009$; SCI $0.137 \pm 0.008)$. Furthermore, gray matter volumes in these regions were not significantly correlated with the degree of SI reorganization and were not significantly different between controls and SCI subjects $[$ mean \pm SEM $($ prob $\times$ vol): right SI: controls $0.48 \pm 0.04$; SCI $0.40 \pm 0.03$; left SI: controls $0.40 \pm 0.07$; SCI $0.37 \pm 0.02$ ].

Comparison of the locations of SI activations during innocuous brushing of the little finger in control and SCI subjects and the region of FA change in SCI subjects confirmed that the FA change was located in the region of SI representing the upper body, the region of SI displaying reorganization.

\section{Thalamocortical fiber tract properties}

Although FA values in area of SI representing the little finger were altered in accordance with the degree of SI reorganization, the fiber tract connecting the ventroposterior (VP) thalamus to this SI region was unaffected. That is, the fiber tract connecting the VP thalamus with the SI region representing the little finger displayed no significant difference in FA values in SCI compared with controls (mean \pm SEM FA: right VP-SI tract: controls $0.43 \pm 0.01$; SCI $0.41 \pm 0.02$; left VP-SI tract: controls $0.44 \pm$ 0.01 ; SCI $0.44 \pm 0.02$ ) (Fig. 4). Further, there was no significant correlation between the FA values and the degree of SI reorganization.

\section{Water movement changes in the SI cortex}

Although in SCI subjects there was no change in the properties of thalamocortical fibers, close inspection of the diffusion directions within the region of decreased MD, revealed significant changes in the direction and magnitude of the principle eigenvectors (Fig. 5). In controls, the principal eigenvector was directed anterior-posterior, whereas in SCI subjects it was directed medial-lateral, toward the region that would normally represent the leg. In contrast, in the coronal plane, both control and SCI subjects had principal eigenvectors that were directed approximately perpendicular to the cortical surface.

\section{Discussion}

Over twenty-five years ago, Merzenich et al. (1984) used electrophysiological techniques to show that following digit amputation in the owl monkey, the SI region normally innervated by the now amputated digit is invaded by neighboring SI representations. Consistent with this report and subsequent human studies (Flor et al., 1995; Bruehlmeier et al., 1998), we found that in complete thoracic SCI subjects, the functional representation of the little finger shifted toward the region that would normally receive inputs from the legs (Wrigley et al., 2009). If this functional reorganization resulted exclusively from the unmasking of dormant synapses, one would expect no change in SI anatomy. Instead, our data revealed that SI reorganization following long-standing SCI is associated with a significant change in SI anatomy. We therefore propose that in humans, cortical reorganization results at least in part from changes in cortical anatomy, such as axonal sprouting.

\section{Gray matter loss in the area of sensory loss}

Complete thoracic SCI is associated with SI gray matter loss, the degree of which is correlated with the degree of SI reorganization. Although SCI resulted in gray matter loss in the SI region representing the lower body, this loss was minimized with increased degrees of reorganization, i.e., the greater the invasion by neighboring SI representations, the less gray matter loss in the lower body SI representation. Although these changes appear contradictory, they are consistent with the idea that SI reorganization is associated with physical changes in cortical anatomy. For example, it is possible that a loss of ascending input neurons results in a gray matter volume decrease, which is followed by a physical invasion of neighboring representations, so the greater the SI reorganization, the smaller the gray matter volume loss. Combined, these two mechanisms could result in the findings presented here.

Despite variations in injury levels (T1 to T11), neither gray matter volume, nor the degree of SI reorganization varied with injury level. This may reflect the relatively little thoracic somato- 


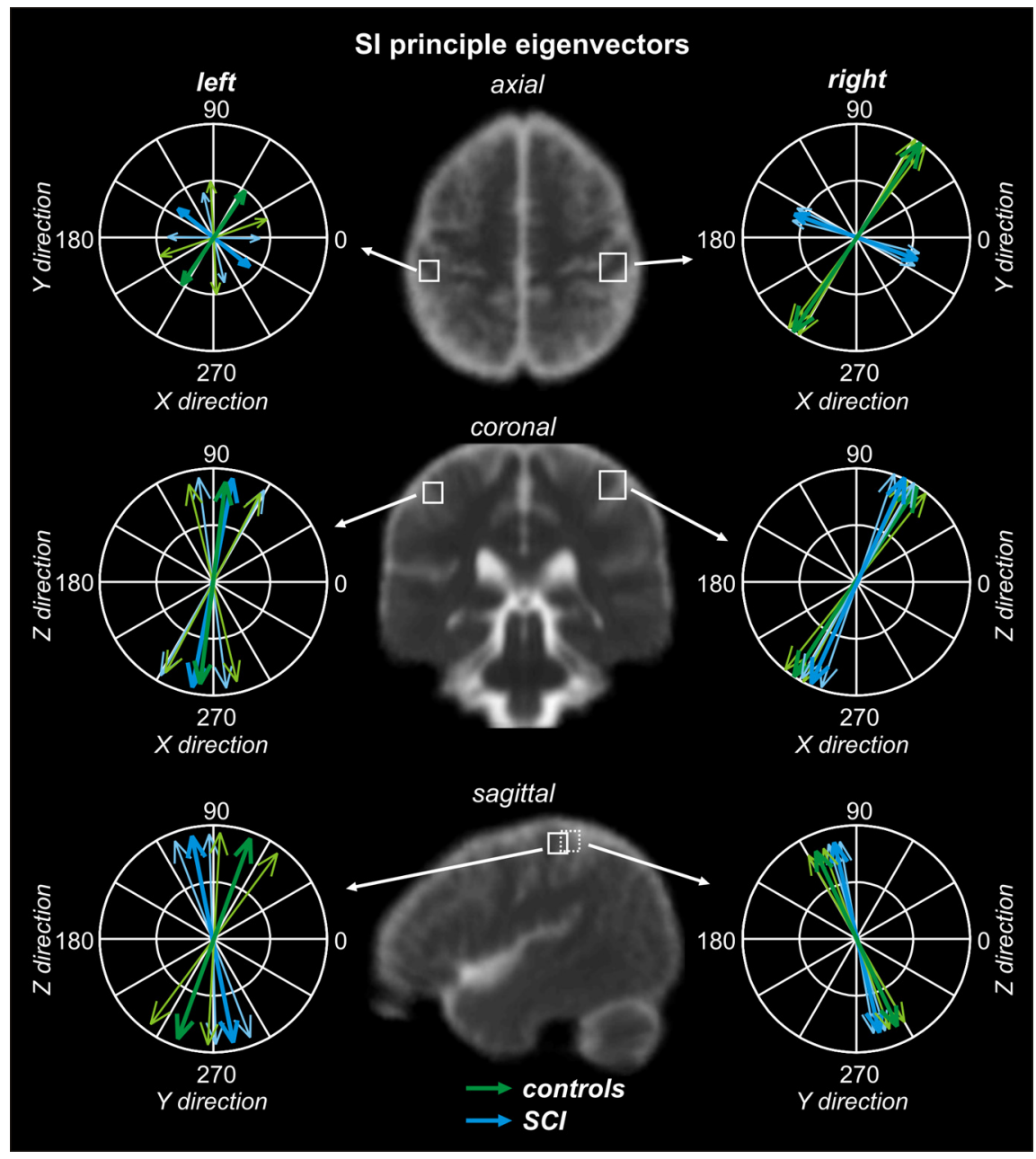

Figure 5. Principal eigenvectors in control and SCI subjects in the SI region in which fractional anisotropy decreased as SI reorganization increased. Mean principle eigenvectors in control (dark green) subjects are directed approximately perpendicular to the cortical surface, In contrast, the principle eigenvectors in SCI subjects (dark blue) are directed more parallel to the cortical surface, i.e., toward the area of sensory deprivation. The SDs of the principle eigenvectors for controls (light green) and SCl subjects (light blue) are also shown.

sensory input, as evidenced by poor two-point discrimination ability (Nolan, 1985). Further, it is unlikely that the change in SI reorganization following SCI resulted from changes in upper limb use, since the most SCI patients reported no change in fine upper limb use following their injuries. We also found that the degree of gray matter loss and SI reorganization did not diminish with age and varied considerably between individuals, despite similar injuries. These findings raise the possibility that the ability of an individual's brain to reorganize and remodel following traumatic injury varies from individual to individual and does not diminish with age.

\section{Latent lateral synapses}

At least some of the cortical reorganization that occurs following SCI may result from the unmasking of dormant synapses. Calford and Tweedale (1988) described rapid SI changes (within a single day) following single digit amputation in flying foxes, and in humans, cortical reorganization can occur within hours following brief limb or digit anesthesia (Rossini et al., 1994; Björkman et al., 2009). This relatively rapid reorganization likely results from disinhibition or facilitation of existing subthreshold inputs onto neighboring cortical representations. The unmasking of latent synapses may be responsible for at least small shifts in cortical organization $(<2-3 \mathrm{~mm})$, and this process may play a role in the cortical reorganization that occurs following complete SCI. However, given that complete SCI results in large-scale SI reorganization, with a shift in the little finger representation of $>13 \mathrm{~mm}$, and the clinical observation that neuropathic SCI pain commonly develops months or even years following injury (Siddall et al., 2003), it is likely that the major mechanism responsible for this large reorganization is the relatively slow growth of lateral connections.

\section{Growth of SI lateral connections}

Nearly two decades ago, Pons et al. (1991) reported a similar degree of SI reorganization in monkeys following unilateral cervical dorsal rhizotomies, with the face representation expanding by up to 14 $\mathrm{mm}$. It was suggested that this extensive expansion was too great to be accounted for by the unmasking of latent connections and overlap of thalamocortical projections (Garraghty and Sur, 1990; Rausell and Jones, 1995). Furthermore, in noninjured animals, horizontal intracortical connections do not exist between areas of SI innervated by the mandibular nerve and those innervated by the second cervical nerve, and limited horizontal interconnections exist between hand and lower jaw/neck SI representations (Manger et al., 1997). Therefore, large cortical shifts that occur following massive sensory losses likely involve multiple mechanisms, which include the relatively rapid unmasking of already existing synapses, but more importantly, the slower growth of new dendrites.

Evidence in other sensory systems also supports the suggestion that axonal sprouting occurs following sensory denervation. Following retinal lesions, axonal sprouting of laterally projecting neurons accompanies topographic remodeling of the visual cortex (Darian-Smith and Gilbert, 1994, 1995). Within the somatosensory system, in macaque monkeys with longstanding, accidental trauma to a forelimb, "thalamocortical projections were at least relatively normal, whereas the changes at the cortical level were immense" (Florence et al., 1998). Furthermore, 5 months after discrete primary motor cortex lesions in primates, Dancause et al. (2005) reported that new connections were formed between the ventral premotor cortex and SI, revealing reorganization between different brain systems.

Our data also support the idea that SI cortical reorganization is associated with expansion of cortical lateral connections. Not only were the changes in SI fractional anisotropy localized to the area that represents the little finger, i.e., the area that displayed the greatest reorganization, but thalamocortical fibers were unaffected, although technical limitations may have limited our ability to detect fine changes in thalamocortical fibers (see below, Limitations). Furthermore, the direction of the principal water movement axis changed from being anterior-posterior in the axial plane to lateromedial, suggesting a greater degree of orga- 
nized water movement toward the area representing the lower body. Indeed, if SI reorganization resulted entirely from the unmasking of latent synapses, one would expect no change in anatomy in the SI region displaying functional reorganization.

\section{Subcortical reorganization}

Subcortical structures may also play a role in SI reorganization. Garraghty and Kaas (1991) described thalamic reorganization following sectioning of peripheral afferents, and following dorsal column lesions or arm amputations in adult monkeys, face afferents in the brainstem trigeminal nucleus can grow into the adjacent cuneate nucleus (Jain et al., 2000). In humans with complete SCI, ventral thalamus cells that would normally respond to inputs from upper extremity respond to inputs from the neck and occiput (Lenz et al., 1987), and sensory thalamus stump representations expand in amputees (Davis et al., 1998). Further, Moore et al. (2000) reported in complete SCI patients that referred sensations were associated with activation of both the SI region representing the body region being stimulated and the area SI representing the location of referred sensation, with $2 \mathrm{~cm}$ of nonresponsive cortex in between. Whether changes in subcortical structures underlie the large cortical reorganization reported in this study remains an open question.

\section{SI reorganization and pain}

It is interesting to speculate how this observed SI reorganization contributes to behavior following SCI, particularly as the changes are occurring in a sensory rather than motor area of the cortex. Any observed behaviors are likely to be influenced and complicated by subsequent CNS processing, and thus not surprising is the evidence from previous work that the strongest link between reorganization and behavior is an abnormality of sensory processing and the presence of pain. We and others have shown that the degree of SI reorganization following amputation or SCI is positively correlated with the intensity of ongoing pain (Flor et al., 1995; Wrigley et al., 2009). Further, the finding that the lip representation did not alter suggests that the changes in SI organization following SCI are site specific and stronger in the region close to the site of deafferentation, rather than global in nature.

\section{Limitations}

Although possible, it is unlikely that SI reorganization following SCI resulted from alterations in upper limb use, since the vast majority of SCI patients reported no increase in fine upper limb use following their injury. Furthermore, our data reveal a shift in SI organization (thumb and little finger representations have shifted) rather than a simple expansion of the hand representation, which would have occurred due to somatosensory training.

While the fiber-tracking technique is not suited to detecting small bundles or nerves, due to resolution and noise limitations, the present study assessed primarily large tracts with this approach, which are more reliably detected by the technique; multiple series also improve signal-to-noise ratios (Turner et al., 2003). Crossing and sprouting of fiber groups are not well detected by fiber-tracking techniques, due to partial-volume and resolution factors, so we were unable to detect fine changes in thalamocortical connections. Such limitations are inherent in all current fiber-tracking approaches based on current DTI methodology.

\section{Conclusions}

We have shown that the functional reorganization in SI following spinal cord injury is accompanied by structural changes. Specifically, the functional representation of the little finger shifted toward the region that would normally receive inputs from the legs, with structural indications of axonal growth between these areas.

\section{References}

Allard T, Clark SA, Jenkins WM, Merzenich MM (1991) Reorganization of somatosensory area $3 \mathrm{~b}$ representations in adult owl monkeys after digital syndactyly. J Neurophysiol 66:1048-1058.

Ashburner J, Friston KJ (2000) Voxel-based morphometry-the methods. Neuroimage 11:805-821.

Ashburner J, Friston KJ (2005) Unified segmentation. Neuroimage 26:839-851.

Basser PJ, Pierpaoli C (1996) Microstructural and physiological features of tissues elucidated by quantitative-diffusion-tensor MRI. J Magn Reson B 111:209-219.

Björkman A, Weibull A, Rosén B, Svensson J, Lundborg G (2009) Rapid cortical reorganisation and improved sensitivity of the hand following cutaneous anaesthesia of the forearm. Eur J Neurosci 29:837-844.

Bruehlmeier M, Dietz V, Leenders KL, Roelcke U, Missimer J, Curt A (1998) How does the human brain deal with a spinal cord injury? Eur J Neurosci 10:3918-3922.

Calford MB, Tweedale R (1988) Immediate and chronic changes in responses of somatosensory cortex in adult flying-fox after digit amputation. Nature 332:446-448.

Dancause N, Barbay S, Frost SB, Plautz EJ, Chen D, Zoubina EV, Stowe AM, Nudo RJ (2005) Extensive cortical rewiring after brain injury. J Neurosci 25:10167-10179.

Darian-Smith C, Gilbert CD (1994) Axonal sprouting accompanies functional reorganization in adult cat striate cortex. Nature 368:737-740.

Darian-Smith C, Gilbert CD (1995) Topographic reorganization in the striate cortex of the adult cat and monkey is cortically mediated. J Neurosci 15:1631-1647.

Davis KD, Kiss ZH, Luo L, Tasker RR, Lozano AM, Dostrovsky JO (1998) Phantom sensations generated by thalamic microstimulation. Nature 391:385-387.

Dougherty RF, Ben-Shachar M, Bammer R, Brewer AA, Wandell BA (2005) Functional organization of human occipital-callosal fiber tracts. Proc Natl Acad Sci U S A 102:7350-7355.

Flor H, Elbert T, Knecht S, Wienbruch C, Pantev C, Birbaumer N, Larbig W, Taub E (1995) Phantom-limb pain as a perceptual correlate of cortical reorganization following arm amputation. Nature 375:482-484.

Florence SL, Taub HB, Kaas JH (1998) Large-scale sprouting of cortical connections after peripheral injury in adult macaque monkeys. Science 282:1117-1121.

Friston KJ, Holmes AP, Poline JB, Grasby PJ, Williams SC, Frackowiak RS, Turner R (1995) Analysis of fMRI time-series revisited. Neuroimage 2:45-53.

Garraghty PE, Kaas JH (1991) Functional reorganization in adult monkey thalamus after peripheral nerve injury. Neuroreport 2:747-750.

Garraghty PE, Sur M (1990) Morphology of single intracellularly stained axons terminating in area $3 \mathrm{~b}$ of macaque monkeys. J Comp Neurol 294:583-593.

Jain N, Florence SL, Qi HX, Kaas JH (2000) Growth of new brainstem connections in adult monkeys with massive sensory loss. Proc Natl Acad Sci U S A 97:5546-5550.

Lenz FA, Tasker RR, Dostrovsky JO, Kwan HC, Gorecki J, Hirayama T, Murphy JT (1987) Abnormal single-unit activity recorded in the somatosensory thalamus of a quadriplegic patient with central pain. Pain 31:225-236.

Macey PM, Macey KE, Kumar R, Harper RM (2004) A method for removal of global effects from fMRI time series. Neuroimage 22:360-366.

Manger PR, Woods TM, Muñoz A, Jones EG (1997) Hand/face border as a limiting boundary in the body representation in monkey somatosensory cortex. J Neurosci 17:6338-6351.

Marino RJ, Barros T, Biering-Sorensen F, Burns SP, Donovan WH, Graves DE, Haak M, Hudson LM, Priebe MM; ASIA Neurological Standards Committee 2002 (2003) International standards for neurological classification of spinal cord injury. J Spinal Cord Med 26:S50-S56.

Merzenich MM, Kaas JH, Wall J, Nelson RJ, Sur M, Felleman D (1983) Topographic reorganization of somatosensory cortical areas $3 \mathrm{~b}$ and 1 in adult monkeys following restricted deafferentation. Neuroscience 8:33-55. 
Merzenich MM, Nelson RJ, Stryker MP, Cynader MS, Schoppmann A, Zook JM (1984) Somatosensory cortical map changes following digit amputation in adult monkeys. J Comp Neurol 224:591-605.

Moore CI, Stern CE, Dunbar C, Kostyk SK, Gehi A, Corkin S (2000) Referred phantom sensations and cortical reorganization after spinal cord injury in humans. Proc Natl Acad Sci U S A 97:14703-14708.

Nolan MF (1985) Quantitative measure of cutaneous sensation twopoint discrimination values for the face and trunk. Phys Ther 65: $181-185$.

Pons TP, Garraghty PE, Ommaya AK, Kaas JH, Taub E, Mishkin M (1991) Massive cortical reorganization after sensory deafferentation in adult macaques. Science 252:1857-1860.

Rausell E, Jones EG (1995) Extent of intracortical arborization of thalamocortical axons as a determinant of representational plasticity in monkey somatic sensory cortex. J Neurosci 15:4270-4288.
Rossini PM, Martino G, Narici L, Pasquarelli A, Peresson M, Pizzella V, Tecchio F, Torrioli G, Romani GL (1994) Short-term brain 'plasticity' in humans: transient finger representation changes in sensory cortex somatotopy following ischemic anesthesia. Brain Res 642:169-177.

Siddall PJ, McClelland JM, Rutkowski SB, Cousins MJ (2003) A longitudinal study of the prevalence and characteristics of pain and non-painful phantom sensations in the first five years following spinal cord injury. Pain 103:249-257.

Turner JA, Lee JS, Schandler SL, Cohen MJ (2003) An fMRI investigation of hand representation in paraplegic humans. Neurorehabil Neural Repair 17:37-47.

Wrigley PJ, Press SR, Gustin SM, Macefield VG, Gandevia SC, Cousins MJ, Middleton JW, Henderson LA, Siddall PJ (2009) Neuropathic pain and primary somatosensory cortex reorganization following spinal cord injury. Pain 141:52-59. 